\title{
Removing Thermostat Distortions of Protein Dynamics in Constant- Temperature Molecular Dynamics Simulations
}

\author{
Alan Hicks ${ }^{1}$, Matthew MacAinsh ${ }^{1}$, and Huan-Xiang Zhou ${ }^{1,2, *}$ \\ ${ }^{1}$ Department of Chemistry and ${ }^{2}$ Department of Physics, University of Illinois at Chicago, \\ Chicago, IL 60607, USA
}

Supporting Information 
Table S1 Rotational correlation times (RCTs) of eight proteins from experimental studies, from NVE simulations, and from NVT simulations at three damping constants $\left(0.2,2,20 \mathrm{ps}^{-1}\right)$

\begin{tabular}{|l|c|c|c|c|c|c|}
\hline \multirow{2}{*}{ Protein } & \multirow{2}{*}{$\begin{array}{c}\text { Temperature } \\
\left({ }^{\circ} \mathrm{K}\right)\end{array}$} & EXP & NVE & NVT-0.2 & NVT-2 & NVT-20 \\
\cline { 3 - 7 } & 298 & 1.09 & $1.21 \pm 0.06$ & $1.24 \pm 0.03$ & $1.77 \pm 0.05$ & $5.74 \pm 0.14$ \\
\hline$\theta$-Defensin & 300 & 1.1 & $0.96 \pm 0.02$ & $0.99 \pm 0.02$ & $1.43 \pm 0.03$ & $5.06 \pm 0.02$ \\
\hline Trp-Cage & 283 & 2.6 & $3.39 \pm 0.32$ & $3.36 \pm 0.04$ & $5.06 \pm 0.14$ & $18.75 \pm 0.77$ \\
\hline ShK & 300 & $3.43 \pm 0.03$ & $3.22 \pm 0.12$ & $3.51 \pm 0.03$ & $6.09 \pm 0.12$ & $26.03 \pm 0.37$ \\
\hline GB3 & 298 & 3.5 & $3.92 \pm 0.48$ & $3.96 \pm 0.15$ & $6.82 \pm 0.11$ & $27.98 \pm 0.15$ \\
\hline BPTI & 300 & 4.1 & $4.33 \pm 0.09$ & $4.92 \pm 0.13$ & $8.77 \pm 0.17$ & $39.83 \pm 1.73$ \\
\hline Ubiquitin & 308 & $5.30 \pm 0.13$ & $4.73 \pm 0.12$ & $5.43 \pm 0.17$ & $10.50 \pm 0.55$ & $46.98 \pm 1.93$ \\
\hline RNase T1 & 308 & $5.7 \pm 0.2$ & $5.79 \pm 0.39$ & $6.50 \pm 0.22$ & $13.42 \pm 0.37$ & $66.63 \pm 1.07$ \\
\hline HEWL & & & & & &
\end{tabular}


Table S2 Root-mean-square-errors (RMSEs) of calculated NH longitudinal and transverse relaxation rates and heteronuclear Overhauser effects

\begin{tabular}{|c|c|c|c|c|c|c|c|c|}
\hline & $\theta$-Defensin & Trp-Cage & $\underline{\operatorname{ShK}}$ & GB3 & BPTI & Ubiquitin & RNase T1 & HEWL \\
\hline \multicolumn{9}{|c|}{$R_{1} \operatorname{RMSE}\left(\mathrm{s}^{-1}\right)$} \\
\hline NVE & 0.12 & 0.53 & 0.16 & 0.18 & 0.16 & 0.17 & 0.22 & 0.36 \\
\hline cNVT-0.2 & 0.12 & 0.54 & 0.12 & 0.16 & 0.17 & 0.14 & 0.18 & 0.36 \\
\hline cNVT-2 & 0.11 & 0.49 & 0.13 & 0.15 & 0.16 & 0.14 & 0.18 & 0.35 \\
\hline cNVT-20 & 0.13 & 0.49 & 0.11 & 0.12 & 0.18 & 0.14 & 0.24 & 0.33 \\
\hline \multicolumn{9}{|c|}{$R_{2} \operatorname{RMSE}\left(\mathrm{s}^{-1}\right)$} \\
\hline NVE & -- & 1.2 & 1.2 & 0.33 & 0.96 & 0.55 & 1.5 & 0.63 \\
\hline cNVT-0.2 & -- & 1.2 & 1.2 & 0.36 & 0.92 & 0.60 & 1.3 & 0.55 \\
\hline cNVT-2 & -- & 1.1 & 1.1 & 0.39 & 0.94 & 0.62 & 1.3 & 0.55 \\
\hline cNVT-20 & -- & 1.1 & 1.2 & 0.56 & 0.96 & 0.69 & 1.5 & 0.63 \\
\hline \multicolumn{9}{|c|}{${ }^{1} \mathrm{H}-{ }^{15} \mathrm{~N}$ Het. NOE RMSE } \\
\hline NVE & 0.15 & 0.18 & 0.05 & 0.04 & 0.12 & 0.10 & 0.03 & 0.09 \\
\hline cNVT-0.2 & 0.12 & 0.20 & 0.06 & 0.04 & 0.12 & 0.09 & 0.03 & 0.08 \\
\hline cNVT-2 & 0.13 & 0.26 & 0.05 & 0.05 & 0.12 & 0.08 & 0.05 & 0.08 \\
\hline cNVT-20 & 0.19 & 0.25 & 0.05 & 0.05 & 0.10 & 0.20 & 0.06 & 0.09 \\
\hline
\end{tabular}




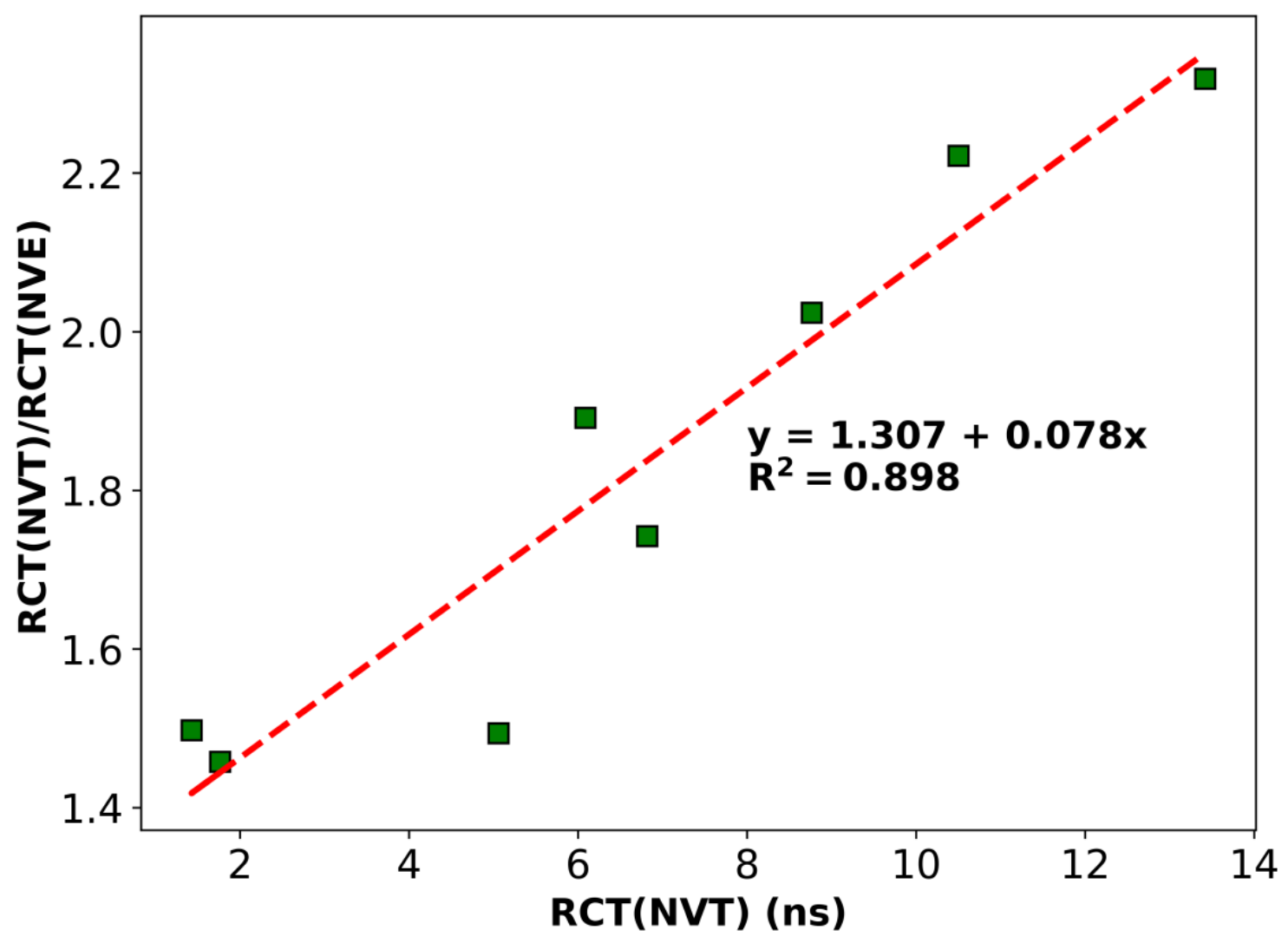

Figure S1. The time dilation by a Langevin thermostat grows with protein size. Time dilation is measured by the ratio between the rotational correlation times (RCTs) calculated from NVT and NVE simulations; protein size is measured by RCT. Each data point is for one of the eight proteins in Fig. 1. NVT simulations were regulated by a Langevin thermostat at $\zeta=2 \mathrm{ps}^{-1}$. 


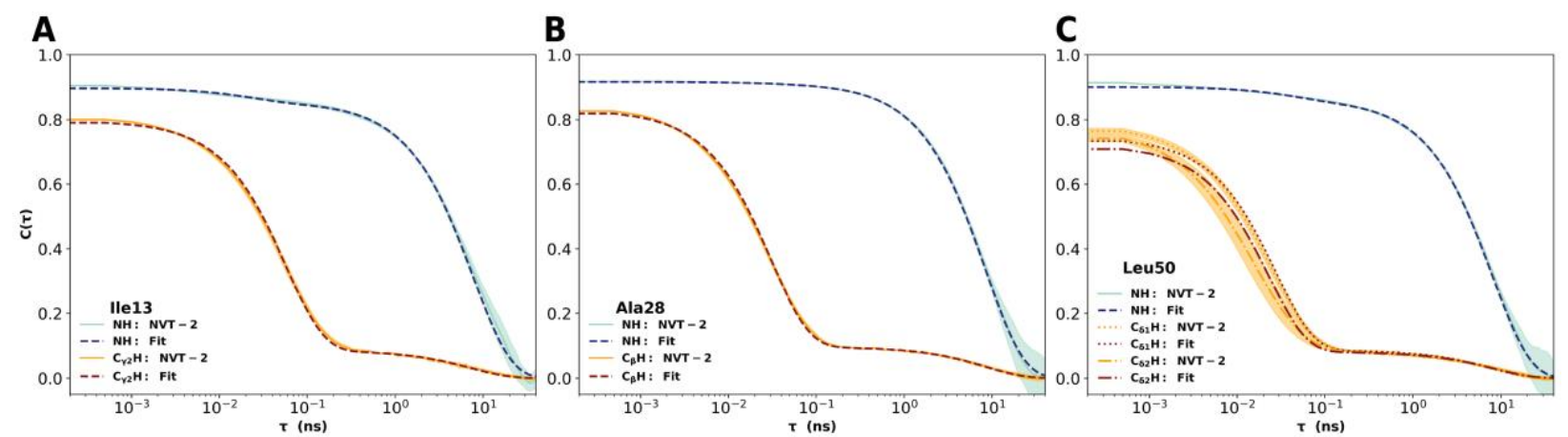

Figure S2. Backbone NH and side-chain methyl $\mathrm{CH}$ time correlation functions, and their biexponential fits. Results are from NVT -2 simulations. Three residues from ubiquitin are chosen for illustration. The standard deviations among four replicate simulations are shown as shading. 

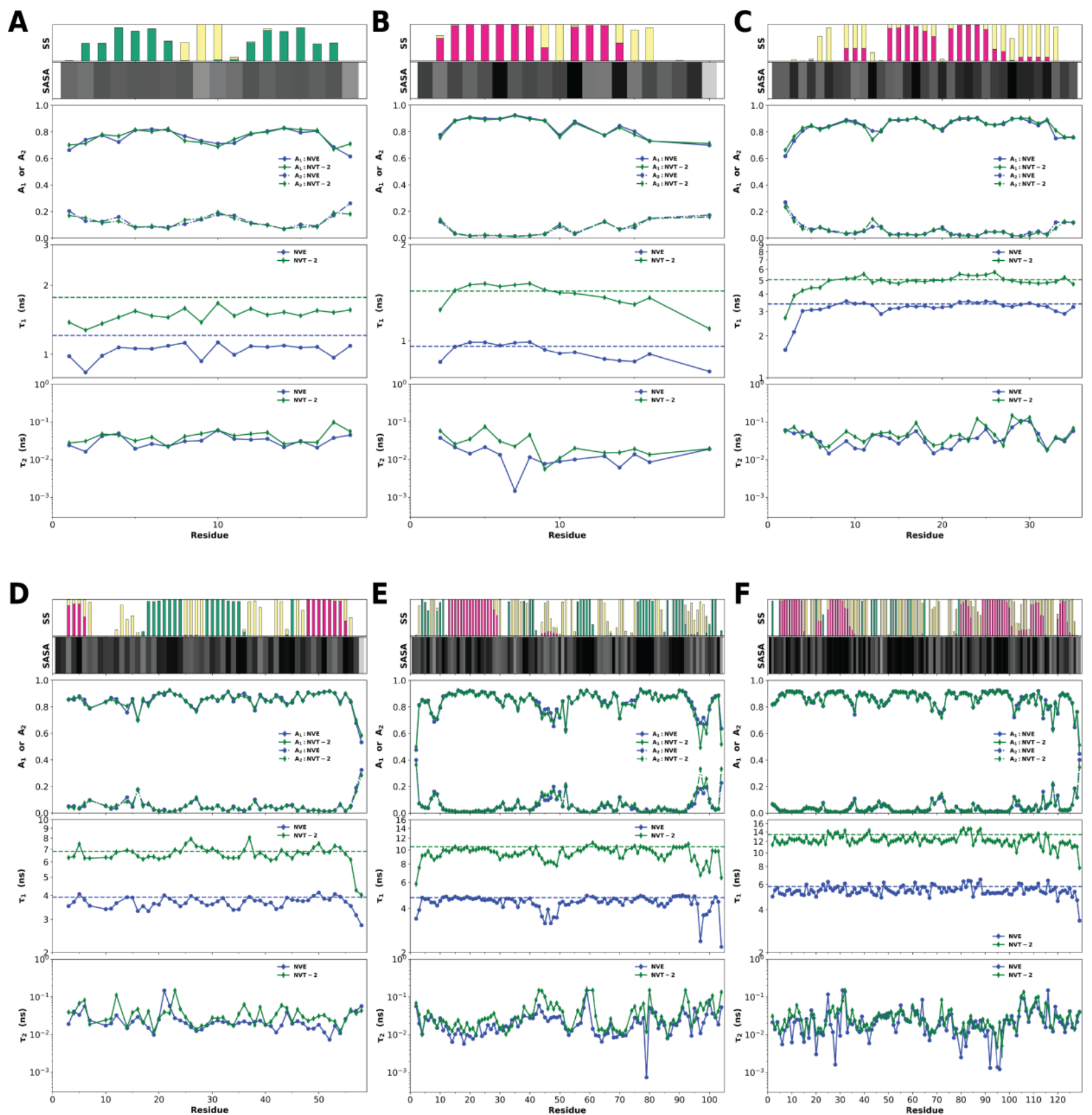

Figure S3. Extension of Fig. 3 to six other proteins. (A) $\theta$-Defensin. (B) Trp-cage. (C) ShK. (D) BPTI. (E) RNase T1. $(F)$ HEWL. In each panel, from top to bottom, the following items are displayed: secondary structure (SS); percent of solvent exposure; $A_{1}$ and $A_{2}$ amplitudes of the bi-exponential fits; $\tau_{1}$ time constant, with rotational correlation times displayed as dashed horizontal lines; $\tau_{2}$ time constant. 

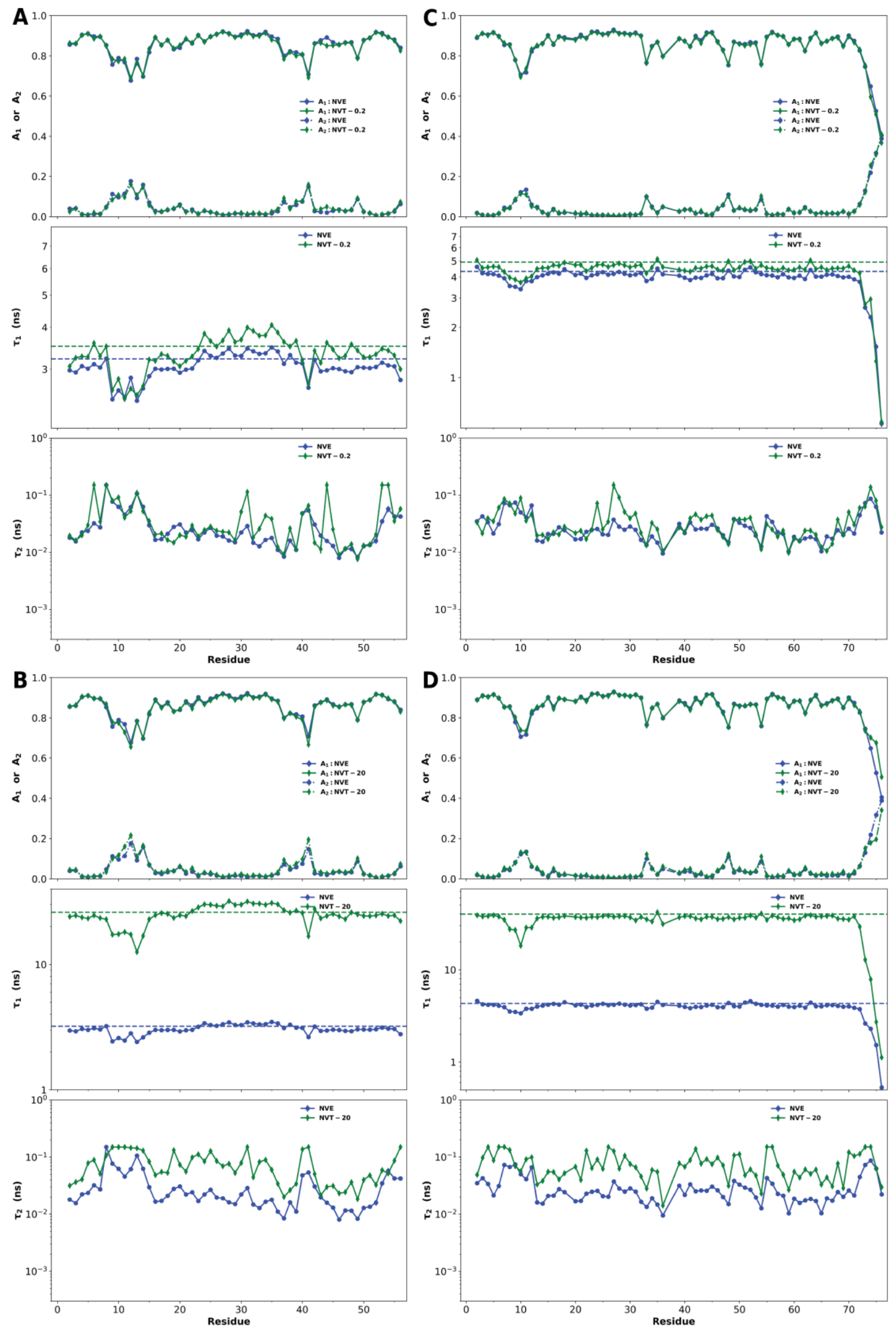

Figure S4. Extension of Fig. 3 to NVT simulations at $\zeta=0.2$ and $20 \mathrm{ps}^{-1}$. (A\&B) GB3. (C\&D) Ubiquitin. 

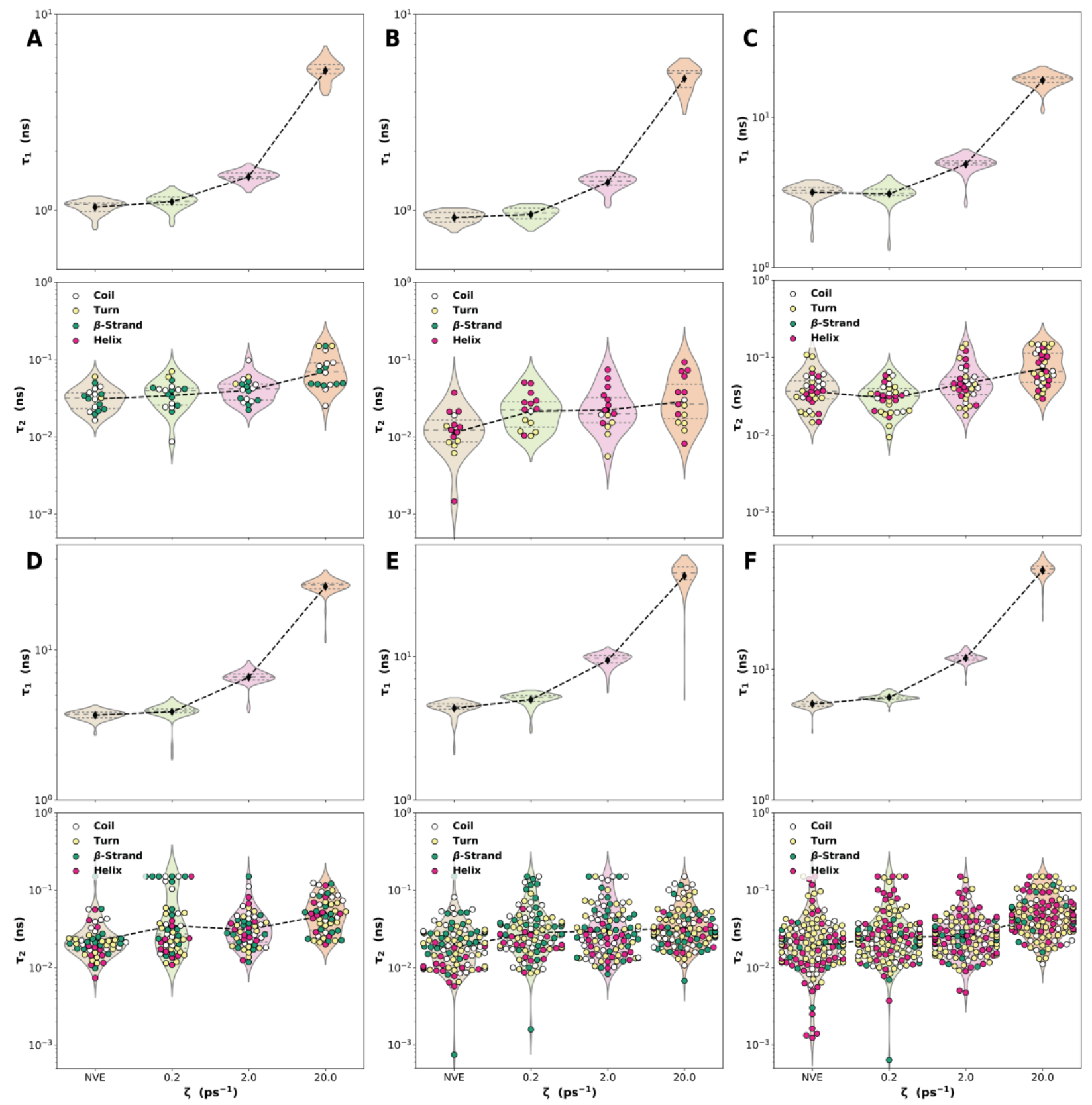

Figure S5. Extension of Fig. 4 to six other proteins. (A) $\theta$-Defensin. (B) Trp-cage. (C) ShK. (D) BPTI. (E) RNase T1. (F) HEWL. 

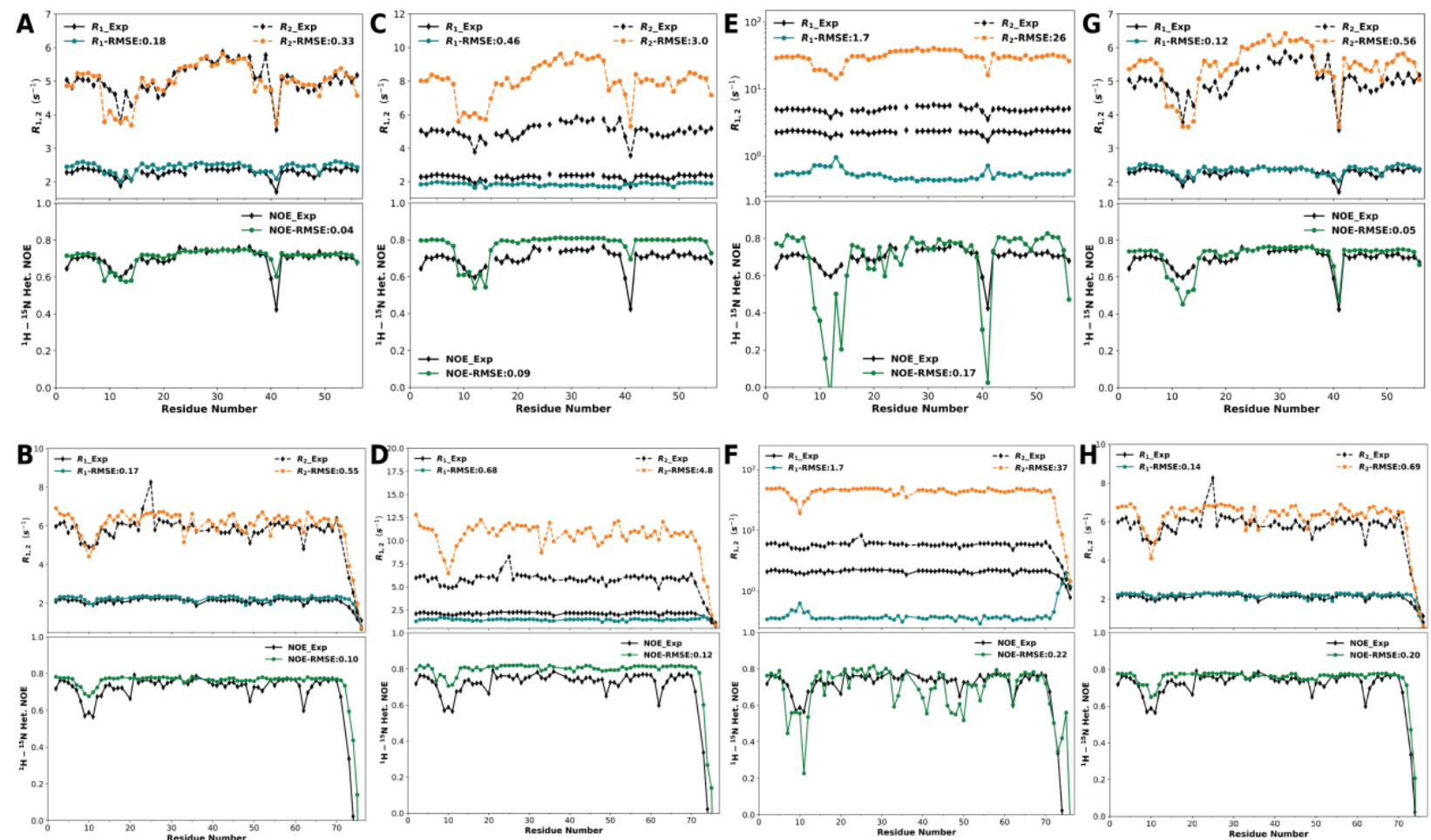

Figure S6. Comparison of backbone NH NMR relaxation properties predicted by NVE and NVT simulations against experimental data. $(A),(C),(E), \&(G)$ are for GB3 and $(B),(D),(F), \&(H)$ are for ubiquitin. $(A) \&(B)$ NVE results. $(C) \&(D)$ NVT results at $\zeta=2 \mathrm{ps}^{-1}$. (E) \& (F) NVT results at $\zeta=20 \mathrm{ps}^{-1}$. $(G) \&(H)$ Corrected NVT results at $\zeta=20 \mathrm{ps}^{-1}$. In each panel, results from MD simulations are shown in color, while experimental data are shown in black. 

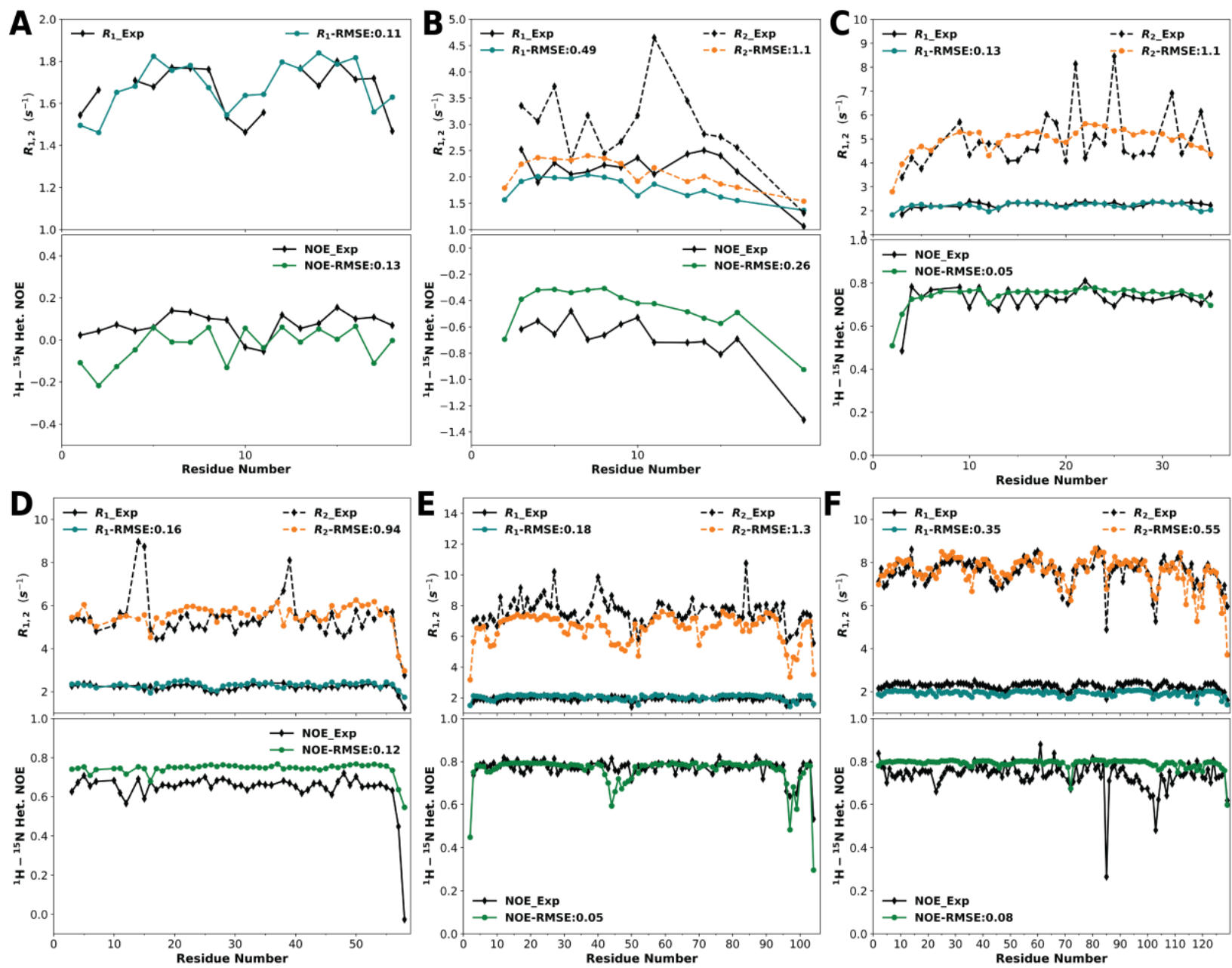

Figure S7. Extension of Fig. 6 to six other proteins. (A) $\theta$-Defensin. (B) Trp-cage. (C) ShK. (D) BPTI. (E) RNase T1. (F) HEWL. 

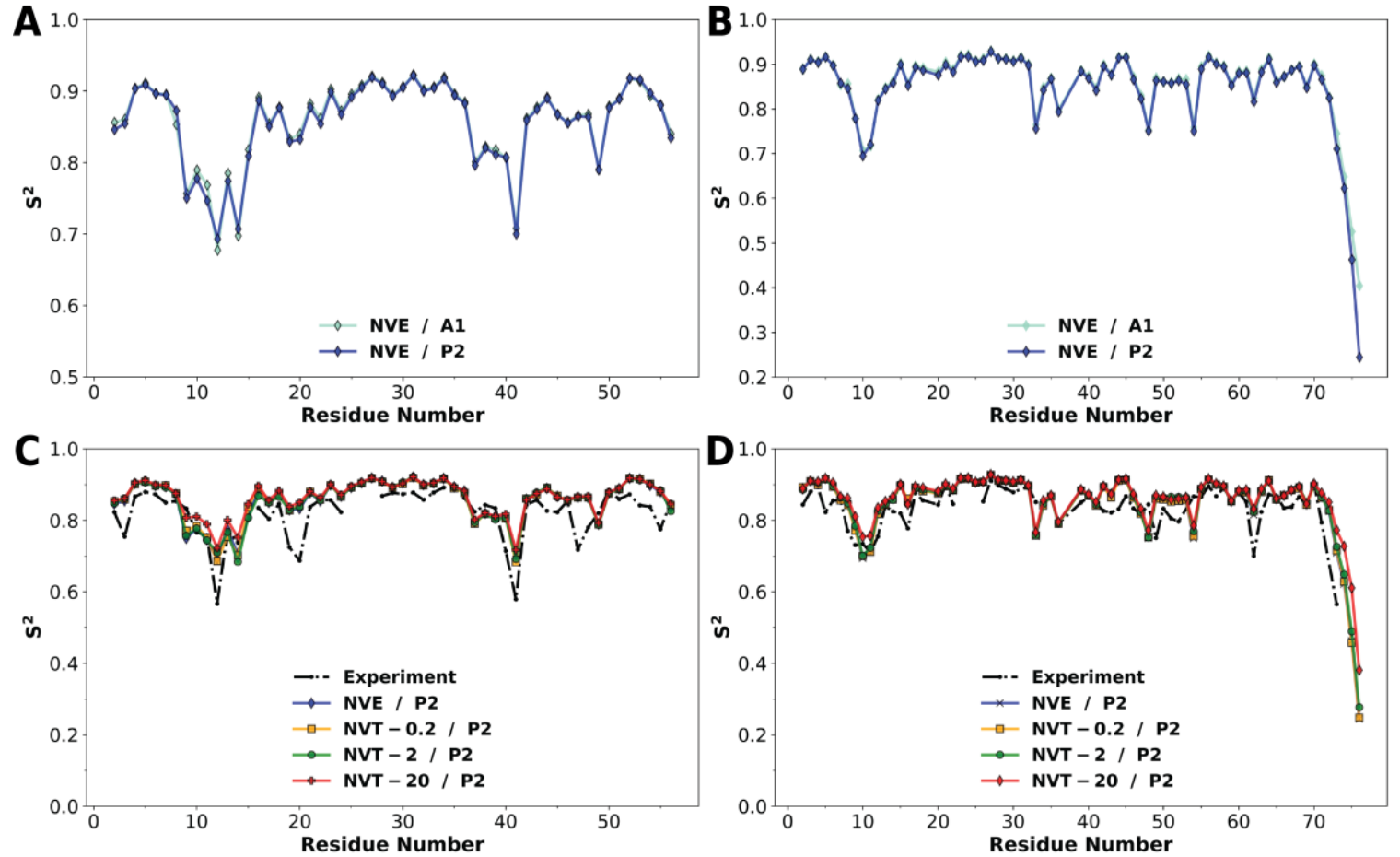

Figure S8. Backbone NH order parameters, $S^{2}$. $(A) \&(C)$ are for GB3 and $(B) \&(D)$ are for ubiquitin. $(A) \&(B)$ Comparison of order parameters from the $\mathrm{A} 1$ and $\mathrm{P} 2$ methods. $(C) \&(D)$ Comparison of order parameters calculated by the P2 method among NVE, NVT -0.2 , NVT -2 , and NVT - 20 simulations, and also against experimental data. The P2 method used 1-ns blocks for structural alignment. 


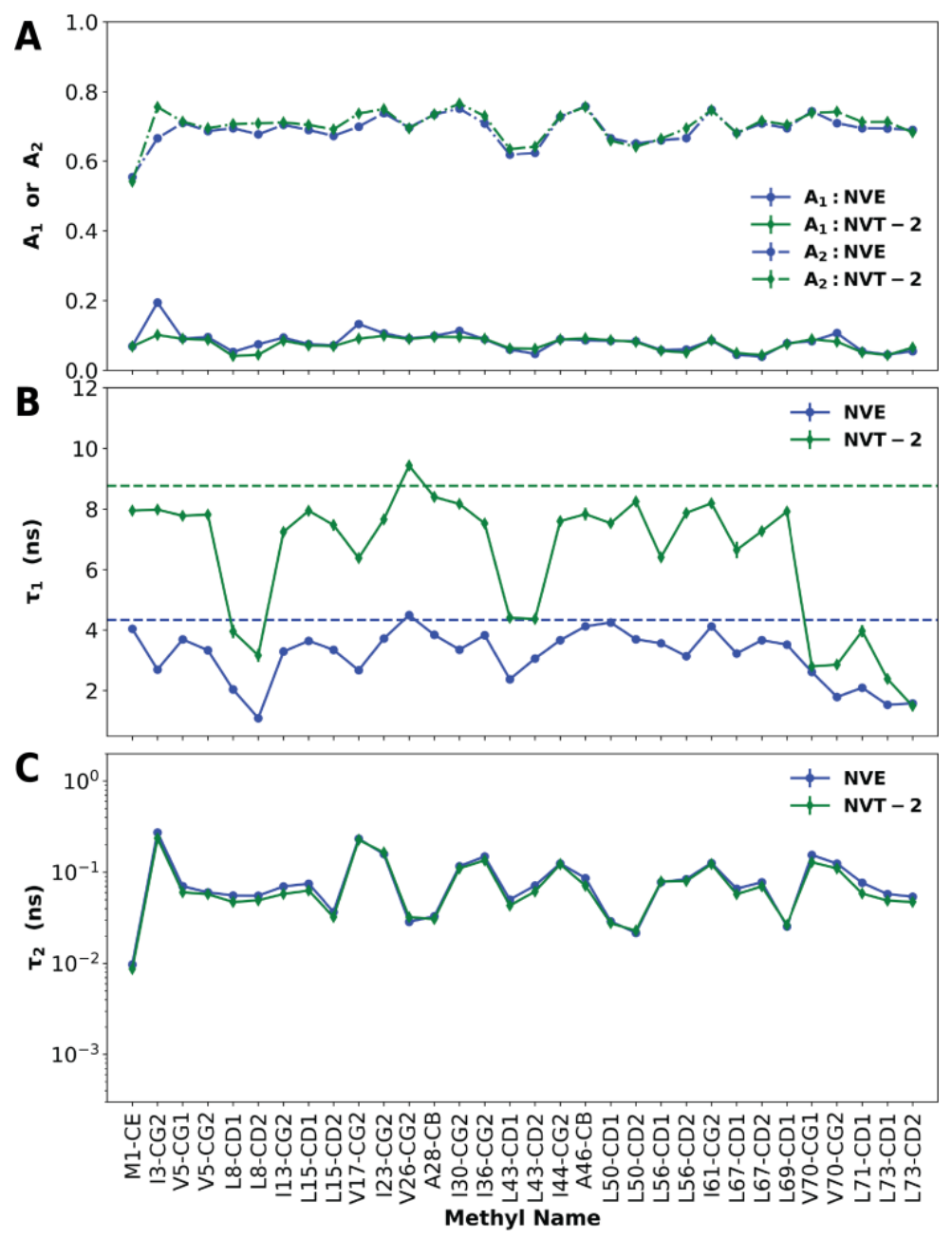

Figure S9. Parameters of bi-exponential fits for ubiquitin methyl $\mathrm{CH}$ time correlation functions. Results for NVE and NVT (at $\zeta=2 \mathrm{ps}^{-1}$ ) simulations are shown. (A) Amplitudes $A_{1}$ and $A_{2}$ of the bi-exponential fits. $(B)$ Time constant $\tau_{1}$. The rotational correlation times calculated from these simulations are displayed as dashed horizontal lines. (C) Time constant $\tau_{2}$. 

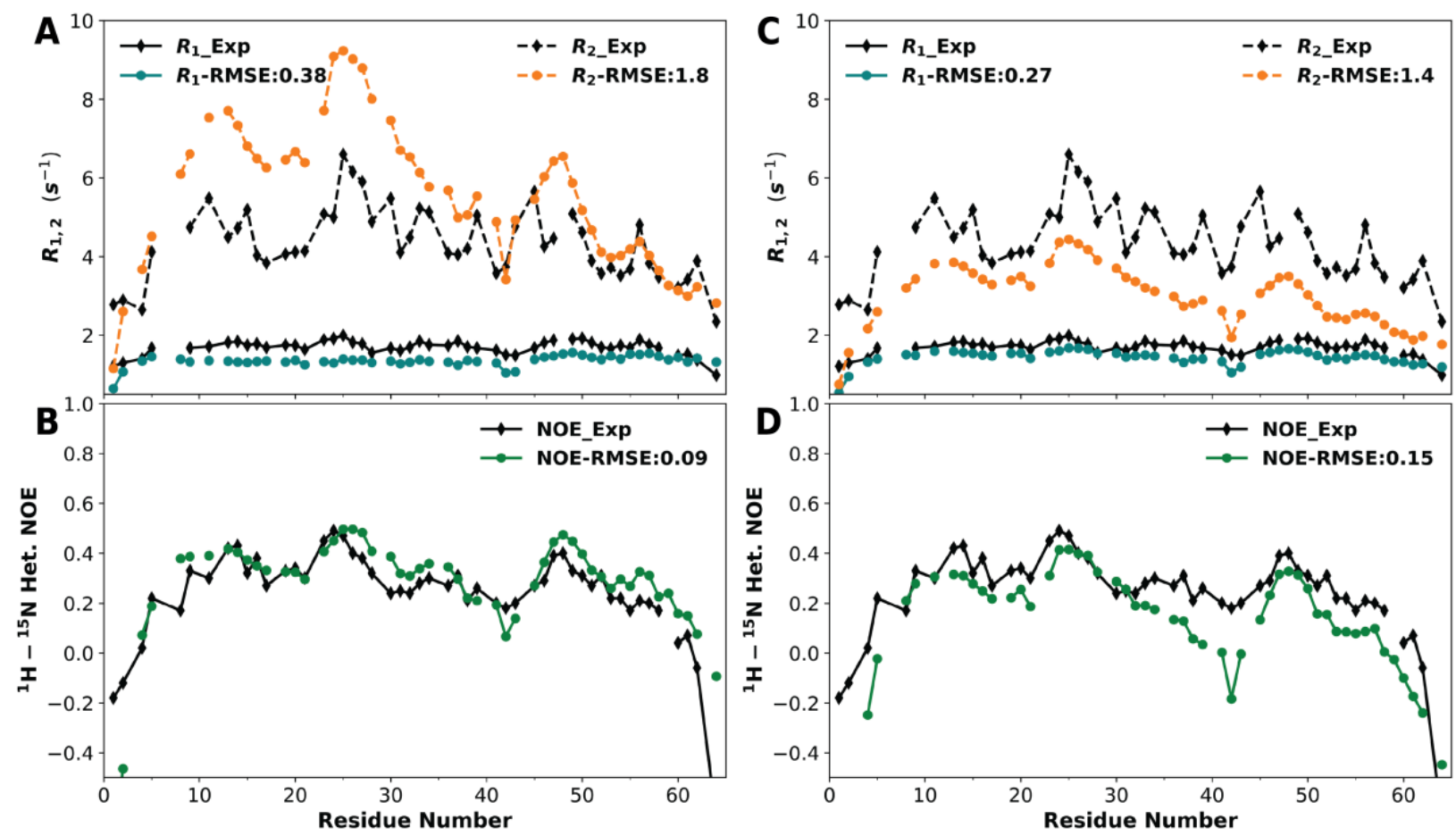

Figure S10. Comparison of backbone NH NMR relaxation properties calculated from NVT simulations of ChiZ against experimental data. $(A) \&(B)$ Results directly from NVT (at $\zeta=3$ $\mathrm{ps}^{-1}$ ) simulations shown in color; experimental data shown in black. $(C) \&(D)$ The same comparison but for simulation results after time-contraction correction. 
A
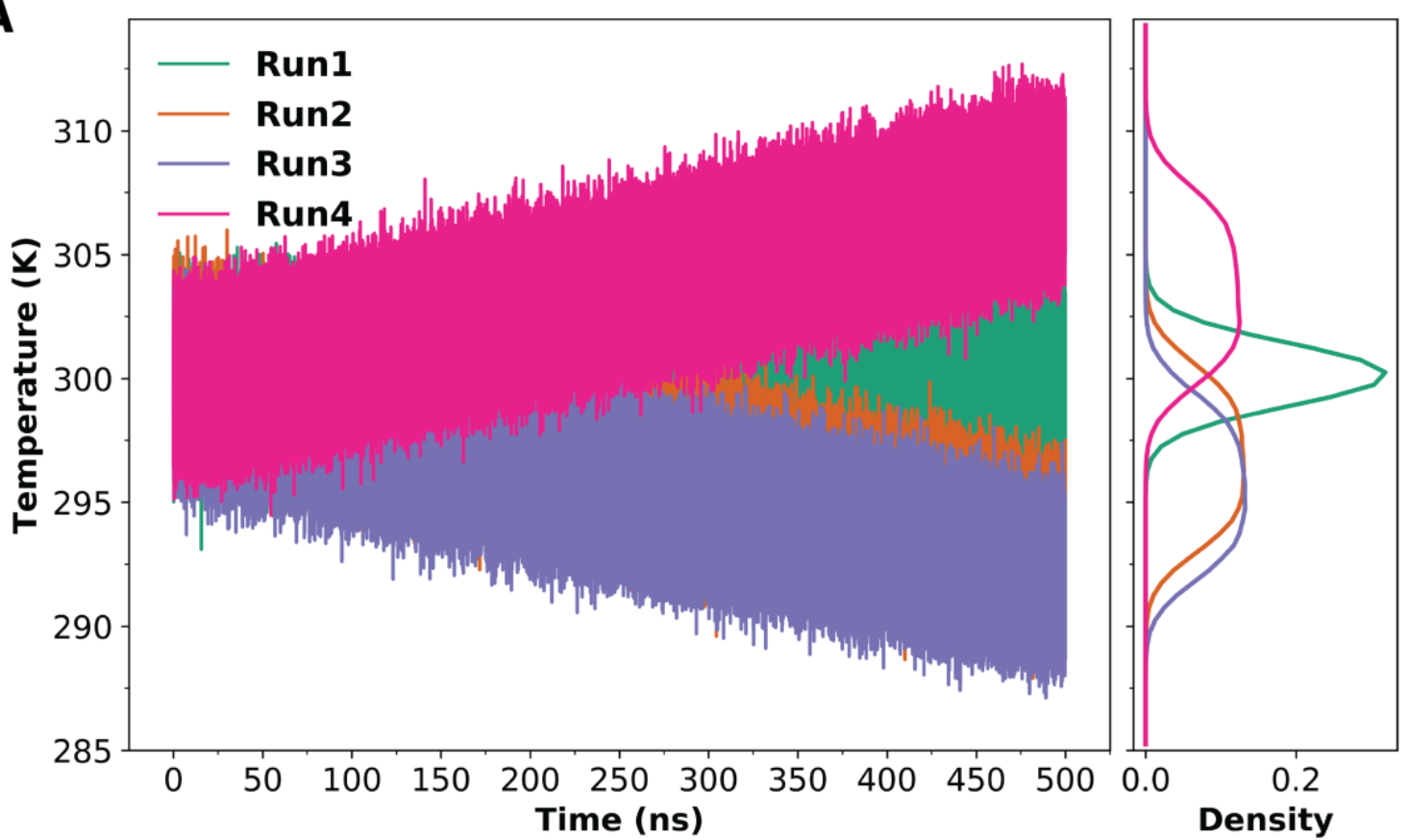

B

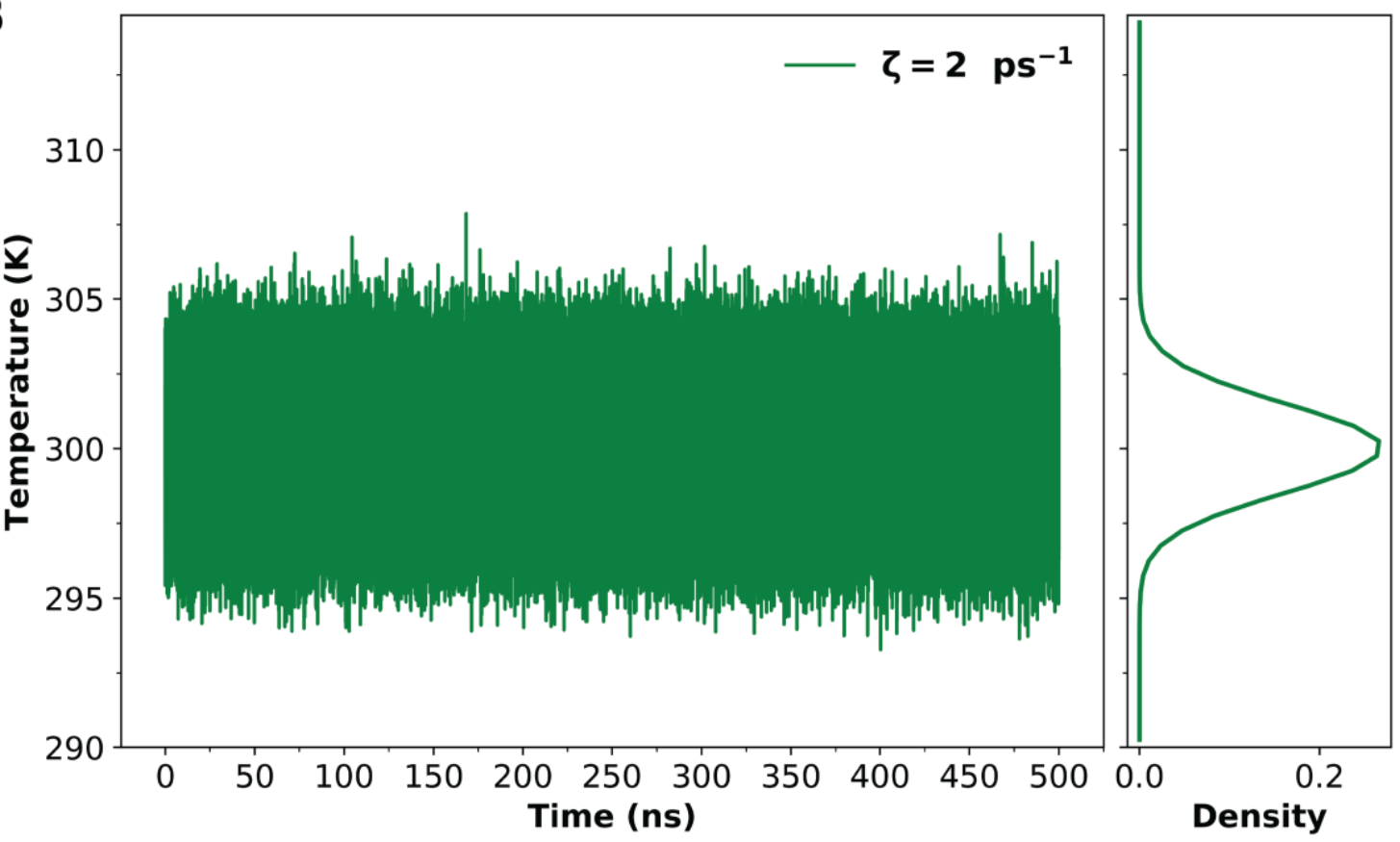

Figure S11. Temperature drift in NVE simulations and temperature regulation by a Langevin thermostat at $\zeta=2 \mathrm{ps}^{-1}$. (A) Time traces of temperature in four replicate NVE runs. Temperature is stable in run1, but drifts upward in run4 and downward in run2 and run3. (B) A representative time trace of temperature in a Langevin simulation, showing stable temperature. 

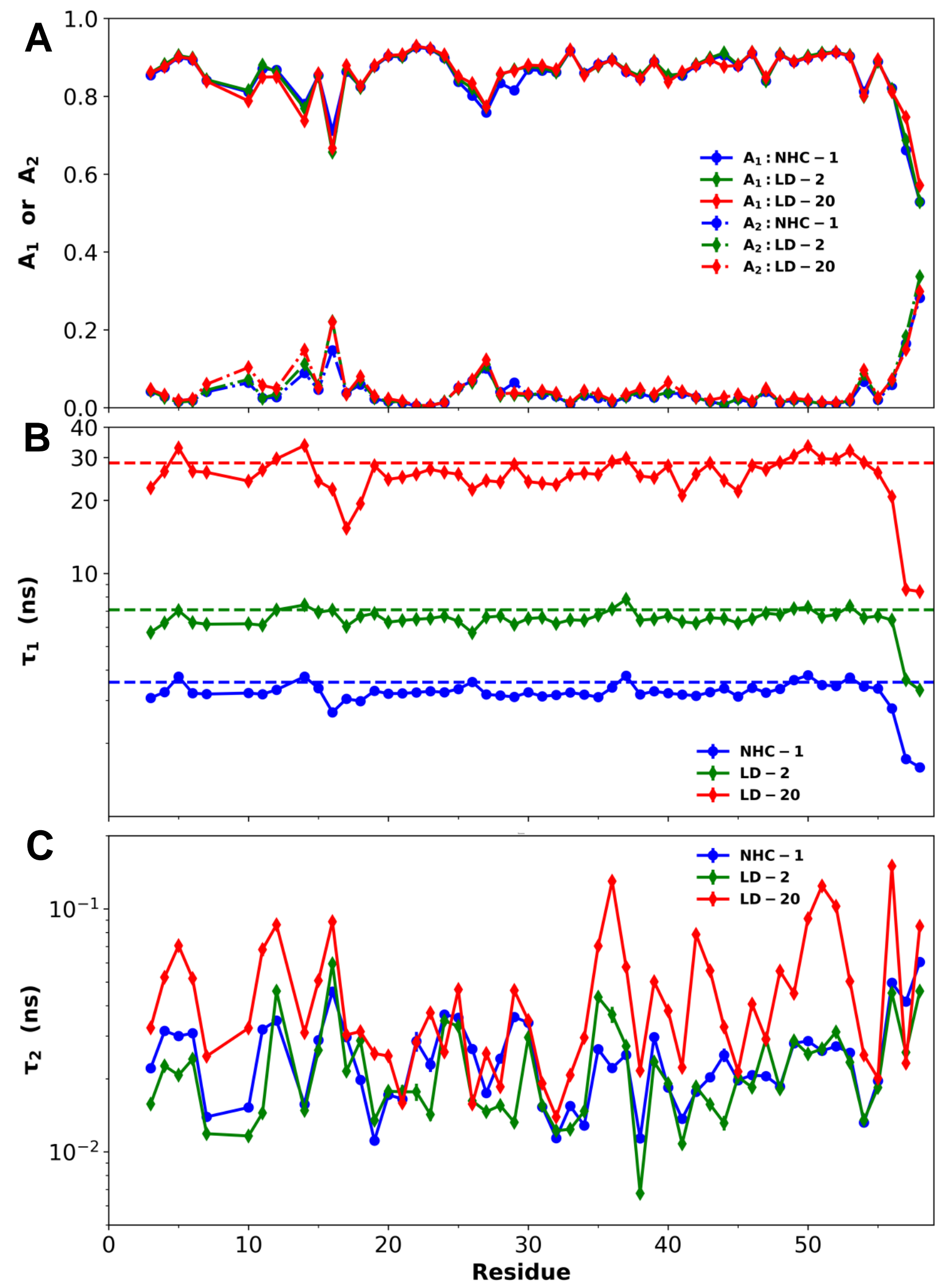

Figure S12. Parameters of bi-exponential fits for NH time correlation functions of BPTI in simulations with the Langevin thermostat (LD -2 or -20 , with $\zeta=2$ or $20 \mathrm{ps}^{-1}$ ) or Nose- 
Hoover chain thermostat (NHC - 1, with relaxation time at $1 \mathrm{ps}$ and chain length at 10$)$. All simulations were run in the GROMACS 2020.6 package (because of the availability of the NHC thermostat), with force fields and other settings copying those in the AMBER simulations. $(A)$ Amplitudes $A_{1}$ and $A_{2}$ of bi-exponential fits, well conserved among all the simulations. $(B)$ Time constant $\tau_{1}$. The rotational correlation times (RCTs) calculated from these simulations are displayed as dashed horizontal lines. The RCTs of the LD - 2 and LD - 20 simulations are 7.09 \pm 0.11 and $28.51 \pm 1.09 \mathrm{~ns}$, very close to the counterparts in AMBER (Table S1). The RCT of the NHC -1 simulations is $3.57 \pm 0.14$, which appears to be mostly free of thermostat distortions. (C) Time constant $\tau_{2}$. Among the 53 backbone amides in BPTI, $\tau_{2}$ values of 33 (or about two thirds of the amides) in the NHC -1 simulations were higher than those in the LD -2 simulations. On average, the NHC $-1 \tau_{2}$ values are higher by 2.4 ps than the LD -2 counterparts for the 53 amides. Because internal motions are already slowed down in LD - 2 simulations, the higher NHC $-1 \tau_{2}$ values can only mean even greater slowdown. When the NHC thermostat relaxation time was increased by 10 -fold or decreased by 10 -fold, the difference in average $\tau_{2}$ between NHC and LD -2 simulations decreased to 1.7 ps and increased to $3.8 \mathrm{ps,}$ respectively. The dependence on thermostat relaxation time confirms a systematic effect of the NHC thermostat. 